\title{
Clinical Significance of the Augmentation Index in Patients with Preserved Kidney Function
}

\author{
Takashi Hitsumoto
}

Hitsumoto Medical Clinic, Yamaguchi

\begin{abstract}
Background: The augmentation index (AIx) indicates arterial wave reflection. Clinical studies have shown a relationship between an elevated AIx and cardiovascular disease. This cross-sectional study attempted to clarify the clinical significance of AIx in patients with preserved kidney function.

Patients and Methods: The subjects were 321 patients with preserved kidney function (an estimated glomerular filtration rate $\geq 60 \mathrm{~mL} / \mathrm{min} / 1.73 \mathrm{~m} 2$ and normoalbuminuria) but with no history of cardiovascular events. The AIx was determined in the radial artery by means of tonometry, and the relationships of the AIx to kidney function and markers of atherosclerosis were examined.

Results: A significant positive correlation $(\mathrm{r}=0.30 ; \mathrm{p}<0.001)$ was found between the AIx and the urinary albumin concentration. The AIx showed a significant positive correlation $(\mathrm{r}=$ $0.28 ; \mathrm{p}<0.001$ ) with the serum high-sensitivity C-reactive protein concentration, as a marker of inflammation; with the urinary 8-iso-prostaglandin $\mathrm{F} 2 \alpha$ concentration $(\mathrm{r}=0.31 ; \mathrm{p}<0.001)$, as a marker of oxidative stress; and with the cardio-ankle vascular index $(r=0.17 ; \mathrm{p}<0.01)$, as a marker of systemic arterial stiffness. Multiple regression analysis indicated that the urinary 8iso-prostaglandin $\mathrm{F} 2 \alpha$ concentration $(\mathrm{t}=5.1 ; \mathrm{p}<0.001)$, serum high-sensitivity $\mathrm{C}$-reactive protein concentration $(\mathrm{t}=4.9 ; \mathrm{p}<0.001)$, and urinary albumin concentration $(\mathrm{t}=3.6 ; \mathrm{p}<0.01)$ were independent variables for AIx after adjustment.
\end{abstract}

Conclusion: These findings indicate that the AIx reflects inflammation, oxidative stress, and the urinary albumin concentration in patients with preserved kidney function.

(J Nippon Med Sch 2012; 79: 422-429)

Key words: augmentation index, preserved kidney function, inflammation, oxidative stress, urinary albumin concentration

\section{Introduction}

Chronic kidney disease, which is usually diagnosed on the basis of a decreased estimated glomerular filtration rate (eGFR) or the presence of proteinuria/ microalubuminuria or both, is a risk factor for both end-stage renal disease and cardiovascular disease ${ }^{1.2}$. Furthermore, from the perspective of preventive medicine, treatment strategies for kidney disease and atherosclerosis should be considered while kidney function is still normal or in the early phase

Correspondence to Takashi Hitsumoto, Hitsumoto Medical Clinic, 2-7-7 Takezaki-cho, Shimonoseki, Yamaguchi 7500025, Japan

E-mail: thitsu@jcom.home.ne.jp

Journal Website (http://www.nms.ac.jp/jnms/) 

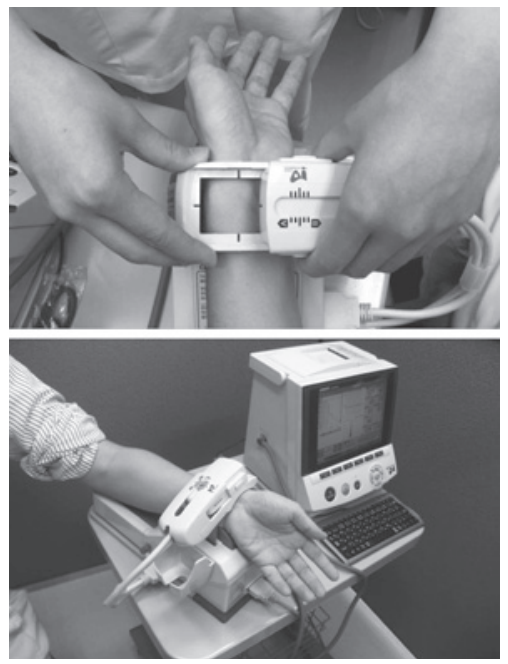

Fig. 1 Measurement of augmentation index

The augmentation index was determined with subjects in the sitting position by means of a commercial device (HEM-9010AI; Omron Healthcare, Kyoto, Japan). The augmentation index is automatically calculated as $\mathrm{P} 2 / \mathrm{P} 1 \times 100 \%$. The P1 and P2 indicate the height of the early and late systolic peaks, respectively.

of kidney disease ${ }^{3}$.

The augmentation index (AIx) indicates arterial wave reflection ${ }^{4}$. Clinical studies have shown that an elevated AIx and an elevated central blood pressure are important predictors of cardiovascular disease ${ }^{5-8}$. However, little is known about the clinical significance of the AIx in patients with preserved kidney function. Here, author examined the relationships of AIx to kidney function and to markers of atherosclerosis to clarify the clinical significance of the AIx in patients with preserved kidney function.

\section{Materials and Methods}

\section{Subjects}

This cross-sectional study was performed at the Hitsumoto Medical Clinic in Shimonoseki from December 2007 through November 2009. The AIx and various clinical variables were analyzed in 321 consecutive patients with lifestyle-related diseases and preserved kidney function. Kidney function was considered to be preserved if the eGFR was at least $60 \mathrm{~mL} / \mathrm{min} / 1.73 \mathrm{~m}^{2}$ and if normoalbuminuria was present (urinary albumin concentrations $<30 \mathrm{mg} / \mathrm{g}$ creatinine). Patients with a history of cardiovascular events, chronic atrial fibrillation, or peripheral artery disease (ankle-brachial index <0.9) were excluded. All patients gave informed consent, and the study protocol was approved by the local ethics committee of Hitsumoto Medical Clinic.

\section{Determination of the AIx and Blood Pressure}

The blood pressure and the AIx were determined in a room with the temperature maintained at $20^{\circ} \mathrm{C}$ to $25^{\circ} \mathrm{C}$. Treatment with antihypertensive drugs was stopped 24 hours or more before measurement. The radial AIx was determined with the subject in the sitting position by means of an applanation tonometry-based device (HEM-9010 AI; Omron Healthcare Co., Ltd., Kyoto, Japan; Fig. 1). This technique has been described in detail previously ${ }^{4,9}$. Briefly, the tonometry sensor unit is a pressure sensor composed of an array of 40 microtransducer elements. When the unit is placed on a patient's wrist, 1 of the 40 sensor elements is automatically selected to obtain optimal radial pressure waveforms. The first and second systolic peaks are automatically detected, and, consequently, the AIx is calculated. The validity and reliability of radial AIx measurement with this method are well established: several studies have indicated a close linear correlation between the radial AIx and the central $\mathrm{AIx}^{4,89}$. In addition, the right brachial blood pressure 
and pulse rate were automatically measured twice with the oscillometric approach. The average of 2 readings was used to determine systolic and diastolic blood pressures and the pulse rate.

\section{Evaluation of Kidney Function}

The eGFR and the urinary albumin concentration were measured as indicators of kidney function. The eGFR was calculated with the adjusted Modification of Diet in Renal Disease study equation, proposed by the working group of the Japanese Chronic Kidney Disease Initiative ${ }^{10}$. The urinary albumin concentration was measured with the latex agglutination method and normalized to urinary creatinine concentration.

\section{Evaluation of Cardiovascular Risk Factors}

Hypertension was diagnosed if the systolic blood pressure was at least $140 \mathrm{mmHg}$, if the diastolic blood pressure was at least $90 \mathrm{mmHg}$, if the subject was receiving antihypertensive treatment, or any combination of these factors. Hyperlipidemia was diagnosed if the serum low-density lipoprotein cholesterol concentrations was at least $140 \mathrm{mg} / \mathrm{dL}$, if serum triglyceride concentrations at least $150 \mathrm{mg} /$ $\mathrm{dL}$, or if the subject was receiving antihyperlipidemic treatment, or any combination of these factors. Diabetes mellitus was diagnosed if the fasting blood glucose levels were at least $126 \mathrm{mg} / \mathrm{dL}$, if the subject had a history of diabetes mellitus or was receiving antidiabetic treatment, or any combination of these factors. Obesity was estimated by the body-mass index. Smoking was considered positive if subjects smoked cigarettes habitually at the start of the study. Blood samples were collected from the antecubital vein in the morning after 12 hours of fasting. Concentrations of serum lipids, plasma glucose, plasma insulin, and serum highsensitivity C-reactive protein (hs-CRP) were subsequently measured. Total cholesterol and triglyceride concentrations were measured with standard enzymatic methods. The high-density lipoprotein cholesterol concentration was measured by means of selective inhibition. The concentration of low-density lipoprotein cholesterol was calculated with Friedewald's formula ${ }^{11}$. The glucose concentration was measured with the glucose oxidase method, and insulin levels were measured with an enzyme immunoassay. The hemoglobin A1c was measured with standard laboratory procedures. To measure insulin resistance, the homeostatic model assessment of insulin resistance (HOMA-IR) was used as follows ${ }^{12}$ : HOMA-IR=fasting glucose concentration $[\mathrm{mg} / \mathrm{dL}] \times$ fasting insulin concentration $[\mu \mathrm{g} / \mathrm{mL}] / 405$. The hs-CRP concentration was measured with high-sensitivity, latex-enhanced immunonephelometrics. The urinary 8-iso-prostaglandin $\mathrm{F} 2 \alpha$ concentration was also measured, as an oxidative stress marker, with an enzyme immunoassay in urine samples and normalized to the urinary creatinine concentration.

\section{Evaluation of Arterial Wall Stiffness}

Systemic arterial wall stiffness was evaluated with the cardio-ankle vascular index (CAVI). The CAVI was determined with a VaSera CAVI instrument (Fukuda Denshi Co. Ltd., Tokyo, Japan) and methods described previously 13 . The average coefficient of variation of the CAVI is less than $5 \%$, which is sufficiently small for clinical usage and indicates that CAVI has good reproducibility ${ }^{13}$.

\section{Statistical Analysis}

A commercially available statistical software program (StatView-J 5.0; Hulinks Inc., Tokyo, Japan) was used for all statistical analyses. Continuous variables were expressed as means \pm standard deviations. Between-group comparisons for continuous variables were performed with Student's t-test. The correlation coefficient was estimated with Spearman's rank correlation analysis. Multivariate analysis was performed with multiple regression analysis. A p value of $<0.05$ was considered to indicate statistical significance.

\section{Results}

Baseline clinical characteristics are shown in Table 1. The mean AIx was $86 \%$, but the AIx was widely distributed, from $46 \%$ to $130 \%$. The mean eGFR was $76 \mathrm{~mL} / \mathrm{min} / 1.73 \mathrm{~mm} 2$. The relationships of AIx with various clinical variables and 
Table 1 Baseline clinical characteristics

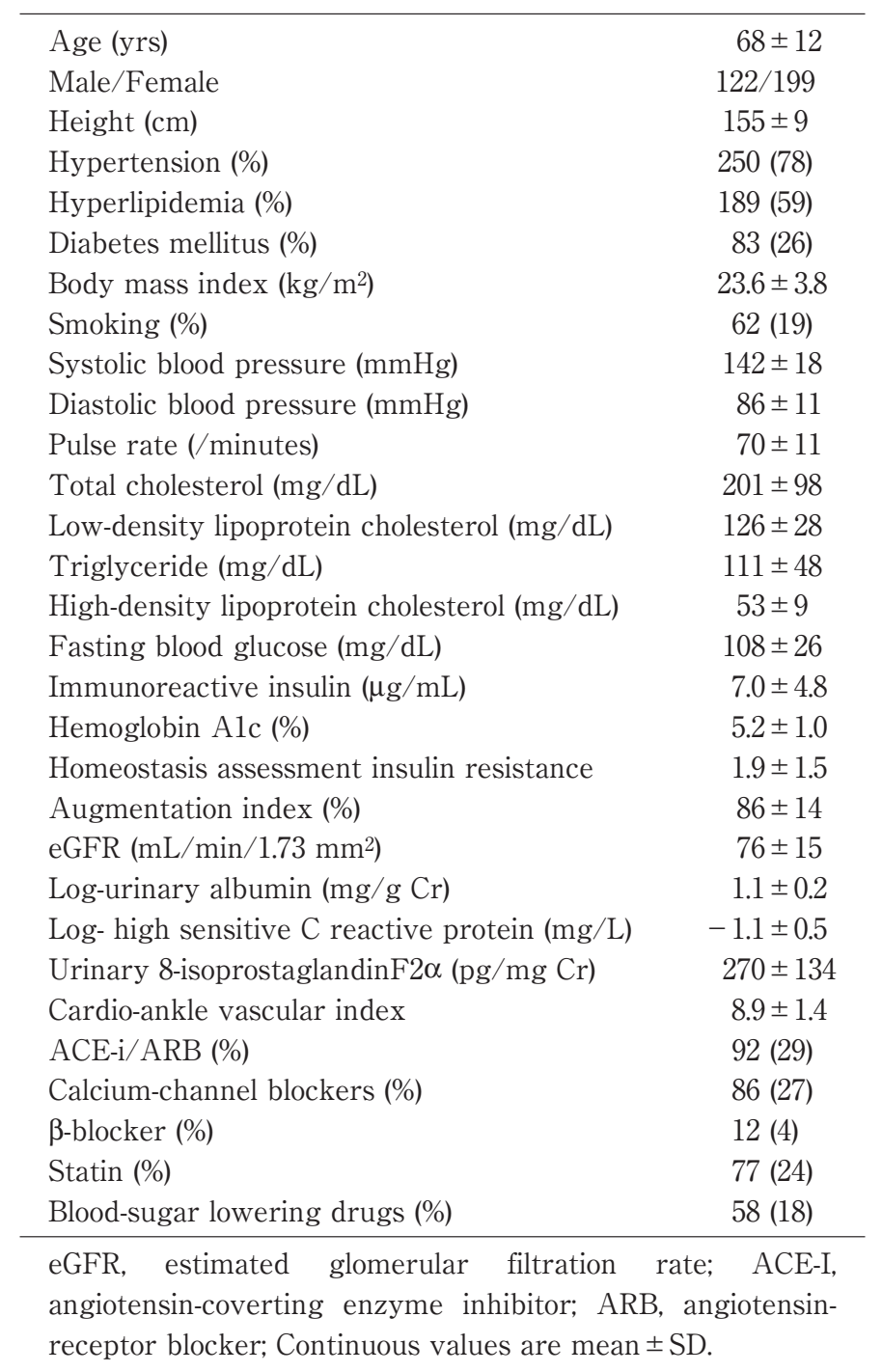

cardiovascular risk factors are shown in Table 2 The AIx showed significant positive correlations with age, systolic blood pressure, the hs-CRP concentration, the urinary 8-iso-prostagrandin F2 $\alpha$ concentration, the CAVI, and the urinary albumin concentration. Conversely, the AIx showed significant negative correlations with the male sex, height, pulse rate, and the eGFR. The AIx showed no significant correlations with the serum lipid, blood glucose, or blood insulin concentration; the hemoglobinA1c; the HOMA-IR; or cardiovascular risk factors, such as hypertension, hyperlipidemia, diabetes mellitus, and smoking habits. The AIx showed no significant relations with medications, such as antihypertensive drugs, statins, and bloodsugar-lowering drugs.

To further investigate the relationship of AIx with kidney function and atherosclerosis, multiple regression analysis was performed to evaluate the ability of ten factors (systolic blood pressure, pulse rate, smoking, age, height, gender $($ male $=1$ and female $=0$ ), eGFR, urinary albumin concentration, hsCRP concentration, and urinary 8-isoprostaglandin F2 $\alpha$ concentration) to explain the augmentation index as a subordinate factor. Six factors (gender, urinary 8-iso-prostaglandin F2 $\alpha$ concentration, hsCRP concentration, pulse rate, urinary albumin concentration, and height) were selected as independent variables for AIx (Table 3). However, age, systolic blood pressure, CAVI, and eGFR were not selected. Multiple regression analysis was also performed for sex differences. Five factors (urinary 8-iso-prostaglandin F $2 \alpha$ concentration, hs-CRP concentration, pulse rate, urinary albumin 


\section{T. Hitsumoto}

Table 2 Relationships between augmentation index and various clinical parameters

\begin{tabular}{|c|c|c|}
\hline & $\mathrm{r}$ & $\mathrm{p}$ value \\
\hline Age & 0.21 & $<0.001$ \\
\hline Gender $($ Male $=1$, Female $=0)$ & -0.23 & $<0.001$ \\
\hline Height & -0.24 & $<0.001$ \\
\hline Hypertension $\left(\mathrm{Yes}=1, \mathrm{No}_{\mathrm{o}}=0\right)$ & 0.13 & NS \\
\hline Hyperlipidemia $\left(\mathrm{Yes}=1, \mathrm{No}_{0}=0\right)$ & 0.11 & NS \\
\hline Diabetes mellitus (Yes $=1, \mathrm{No}_{0}=0$ ) & 0.06 & NS \\
\hline Body mass index & -0.07 & NS \\
\hline Smoking $\left(\mathrm{Yes}=1, \mathrm{No}_{0}=0\right)$ & 0.11 & NS \\
\hline Systolic blood pressure & 0.18 & $<0.01$ \\
\hline Diastolic blood pressure & 0.02 & NS \\
\hline Pulse rate & -0.31 & $<0.001$ \\
\hline Total cholesterol & -0.03 & NS \\
\hline Low-density lipoprotein cholesterol & -0.02 & NS \\
\hline Triglyceride & -0.03 & NS \\
\hline High-density lipoprotein cholesterol & 0.02 & NS \\
\hline Fasting blood glucose & -0.09 & NS \\
\hline Immunoreactive insulin & -0.10 & NS \\
\hline Hemoglobin Alc & -0.09 & NS \\
\hline Homeostasis assessment insulin resistance & -0.09 & NS \\
\hline eGFR & -0.15 & $<0.01$ \\
\hline Log-urinary albumin & 0.30 & $<0.001$ \\
\hline Log- high sensitive $\mathrm{C}$ reactive protein & 0.28 & $<0.001$ \\
\hline Urinary 8-isoprostaglandinF2 $\alpha$ & 0.31 & $<0.001$ \\
\hline Cardio-ankle vascular index & 0.17 & $<0.01$ \\
\hline ACE-i/ARB $\left(Y e s=1, N_{0}=0\right)$ & -0.09 & NS \\
\hline Calcium-channel blockers $(\mathrm{Yes}=1, \mathrm{No}=0)$ & -0.04 & NS \\
\hline$\beta$-blocker $\left(\mathrm{Yes}=1, \mathrm{No}_{0}=0\right)$ & 0.11 & NS \\
\hline Statin $\left(\mathrm{Yes}=1, \mathrm{No}_{0}=0\right)$ & -0.10 & NS \\
\hline Blood-sugar lowering drugs $(\mathrm{Yes}=1, \mathrm{No}=0)$ & 0.05 & NS \\
\hline
\end{tabular}

Abbreviation as in Table 1.

Table 3 Results of multiple regression analysis of augmentation index

\begin{tabular}{lcrc}
\hline & Standard regression coefficient & t value & $\mathrm{p}$ value \\
\hline Acceptable factor & & & \\
Gender (Male=1, Female=0) & -0.25 & -5.2 & $<0.001$ \\
U-8-isoPGF2 $\alpha$ & 0.24 & 5.1 & $<0.001$ \\
Log-hs-CRP & 0.23 & 4.9 & $<0.001$ \\
Pulse rate & -0.21 & -4.5 & $<0.001$ \\
Log-U-Alb & 0.18 & 3.6 & $<0.01$ \\
Height & -0.16 & -3.2 & $<0.01$ \\
\hline
\end{tabular}

$\mathrm{R}^{2}=0.34, \mathrm{~F}$ value $=32.5, \mathrm{p}<0.001,(\mathrm{n}=321)$

concentration, and height) were selected as independent variables for the AIx in both male subjects and female subjects.

\section{Discussion}

The AIx showed significant relationships with markers of kidney function. The AIx also showed significant relationships with markers of 
atherosclerosis, such as the hs-CRP and urinary 8iso-prostaglandin F2 $\alpha$ concentrations, and the CAVI. Multiple regression analysis indicated that urinary albumin, hs-CRP, and urinary 8-iso-prostaglandin F2 $\alpha$ concentrations were independent variables for the AIx as a subordinate factor, following adjustments for gender, height, and pulse rate, which are known to strongly affect the AIx.

Several studies have indicated the importance of a decrease in GFR during cardiovascular events ${ }^{1,2,14}$, and the eGFR is commonly used worldwide to evaluate the stage of chronic kidney disease. In the present study, the AIx showed a significant negative correlation with the eGFR on simple linear regression analysis; however, the correlation coefficient was only -0.15 , and multiple regression analysis indicated that the eGFR was not an independent variable for AIx as a subordinate factor. Therefore, AIx must be considered a variable different from the GFR, and the measurement of both eGFR and AIx would be of benefit when evaluating cardiovascular risk in patients with preserved kidney function.

Microalbuminuria is an important cardiovascular risk factor, and epidemiological studies have revealed that an increase in urinary albumin concentration during the normoalbuminuria phase is also an important risk factor for cardiovascular disease $^{15,16}$. Therefore, from the perspective of preventing cardiovascular disease, urinary albumin concentration should be decreased to the lowest possible level. The urinary albumin concentration is considered to reflect not only glomerular hypertension but also systemic endothelial function ${ }^{17}$.

Conversely, Shirai et al. have reported that the CAVI, which is essentially the stiffness parameter of a long segment of arterial wall, is unaffected by blood pressure at the time of measurement ${ }^{13}$. In the present study, both the urinary albumin concentration and the CAVI showed significant positive correlations with the AIx on simple linear regression analysis; however, multiple regression analysis indicated that the CAVI was not an independent variable for AIx as a subordinate factor, even though urinary albumin concentration was. In fact, several clinical studies have found that the AIx is elevated when arterial wall stiffness increases ${ }^{18}$; however, the present data indicate that the AIx does not reflect arterial wall stiffness in patients without apparent kidney disease. Soga et al. have also reported a close relationship between the AIx and endothelial dysfunction, which was estimated by means of flow-mediated vasodilation in the brachial $\operatorname{artery}^{19}$. Endothelial dysfunction is the first step in the progression to atherosclerosis; furthermore, endothelial dysfunction is independently related to future cardiovascular events. Therefore, AIx could potentially be an important target factor for the prevention of cardiovascular events which is not detectable by evaluation of arterial stiffness in patients with preserved kidney function.

Recent basic and clinical studies have shown that chronic inflammation and oxidative stress in vivo contribute to the development of atherosclerosis ${ }^{20-22}$. In the present study, multiple regression analysis revealed independent associations between the AIx and the hs-CRP concentration, as a marker of inflammation, and with the urinary 8-isoprostaglandin F2 $\alpha$ concentration, as a marker of oxidative stress. Several clinical studies have also found that the serum CRP and isoprostane concentrations have close relationships with the $\mathrm{AIx}^{23,24}$. These results suggest that an increase in inflammation or oxidative stress in vivo plays an important role in increasing the arterial wave reflection. Therefore, early intervention, to decrease inflammation and oxidative stress, could prevent cardiovascular disease resulting from a decrease in the AIx in patients with preserved kidney function.

The AIx is decreased by vasodilators, such as calcium channel blockers, angiotensin II receptor antagonists, and nitrates ${ }^{25,26}$. Conversely, various drugs, including vasodilators and statins, decrease inflammation and oxidative stress in vivo ${ }^{27.28}$. In the present cross-sectional study, AIx showed no significant relation with medications, such as antihypertensive drugs or statins. However, prospective study is warranted to examine the effectiveness of these drugs from the viewpoint of the AIx, inflammation, and oxidative stress; consequently, new applications for these drugs for preventing cardiovascular disease will likely be 
discovered.

The present study had several limitations. Treatment with antihypertensive drugs was stopped 24 hours or more before measurement to avoid influencing AIx. However, 24 hours was not enough to avoid the effects of long-acting drugs, such as amlodipine. The AIx showed significant relationships with various markers of atherosclerosis. However, this was a cross-sectional study in patients with no history of cardiovascular events. Therefore, it remains unclear whether elevation of the AIx is a cardiovascular risk factor in patients with preserved kidney function. Prospective studies involving larger numbers of subjects are necessary to confirm the significance of the AIx as a risk factor for primary cardiovascular events in patients with preserved kidney function, and cut-off values for the AIx should be determined to indentify high-risk patients. Furthermore, an extensive examination of basic and clinical studies investigating the significance of the AIx is required.

In conclusion, results of the present study indicate that the AIx reflects oxidative stress, inflammation, and the urinary albumin concentration in patients with preserved kidney function and suggest that an elevated AIx can be used to predict primary cardiovascular events in patients with preserved kidney function.

\section{Conflicts of interest: None}

\section{References}

1. Coresh J, Selvin E, Stevens LA, et al.: Prevalence of chronic kidney disease in the United States. JAMA 2007; 298: 2038-2047.

2. Yamagata K, Ishida K, Sairenchi T, et al.: Risk factors for chronic kidney disease in a communitybased population: a 10-year follow-up study. Kidney Int 2007; 71: 159-166.

3. Curhan GC: Prediabetes, prehypertension ... is it time for pre-CKD? Clin J Am Soc Nephrol 2010; 5: 557559 .

4. Takazawa K, Kobayashi H, Shindo N, Tanaka N, Yamashina A: Relationship between radial and central arterial pulse wave and evaluation of central aortic pressure using the radial arterial pulse wave. Hypertens Res 2007; 30: 219-228.

5. Williams B, Lacy PS, Thom SM, et al.: CAFE Investigators; Anglo-Scandinavian Cardiac Outcomes Trial Investigators; CAFE Steering Committee and
Writing Committee: Differential impact of blood pressure-lowering drugs on central aortic pressure and clinical outcomes: principal results of the Conduit Artery Function Evaluation (CAFE) study. Circulation 2006; 113: 1213-1225.

6. Roman MJ, Devereux RB, Kizer JR, et al.: Central pressure more strongly relates to vascular disease and outcome than does brachial pressure: the Strong Heart Study. Hypertension 2007; 50: 197-203.

7. London GM, Blacher J, Pannier B, Guérin AP, Marchais SJ, Safar ME: Arterial wave reflections and survival in end-stage renal failure. Hypertension 2001; 38: 434-438.

8. Otsuka T, Kawada T, Ibuki C, Kusama Y: Radial arterial wave reflection is associated with the MEGA risk prediction score, an indicator of coronary heart disease risk, in middle-aged men with mild to moderate hypercholesterolemia. J Atheroscler Thromb 2010; 17: 688-694.

9. Kohara K, Tabara Y, Oshiumi A, Miyawaki K, Kobayashi T, Miki T: Radial augmentation index: a useful and easily obtainable parameter for vascular aging. Am J Hypertens 2005; 18: 11S-14S.

10. Imai E, Horio M, Nitta $K$, et al.: Estimation of glomerular filtration rate by the MDRD study equation modified for Japanese patients with chronic kidney disease. Clin Exp Nephrol 2007; 11: 41-50.

11. Fridewald WT, Levy RI, Fredrickson DS: Estimation of the concentration of low density lipoprotein cholesterol in plasma, without use of the preparative ultracentrifuge. Clin Chem 1972; 18: 499-502.

12. Matthews DR, Hosker JP, Rudenski AS, Naylor BA, Treacher DF, Turner RC: Homeostasis model assessment: insulin resistance and beta-cell function from fasting plasma glucose and insulin concentrations in man. Diabetologia 1985; 28: 412419 .

13. Shirai K, Utino J, Otsuka K, Takata M: A novel blood pressure-independent arterial wall stiffness parameter; cardio-ankle vascular index (CAVI). J Atheroscler Thromb 2006; 13: 101-107.

14. Ninomiya T, Kiyohara Y, Tokuda Y, et al.: Japan Arteriosclerosis Longitudinal Study Group: Impact of kidney disease and blood pressure on the development of cardiovascular disease: an overview from the Japan Arteriosclerosis Longitudinal Study. Circulation 2008; 118: 2694-2701.

15. Gerstein HC, Mann JF, Yi Q, et al.: HOPE Study Investigators: Albuminuria and risk of cardiovascular events, death, and heart failure in diabetic and nondiabetic individuals. JAMA 2001; 286: 421-426.

16. Arnlöv J, Evans JC, Meigs JB, et al.: Low-grade albuminuria and incidence of cardiovascular disease events in nonhypertensive and nondiabetic individuals: the Framingham Heart Study. Circulation 2005; 112: 969-975.

17. Stehouwer CD, Nauta JJ, Zeldenrust GC, Hackeng WH, Donker AJ, den Ottolander GJ: Urinary albumin excretion, cardiovascular disease, and endothelial dysfunction in non-insulin-dependent diabetes mellitus. Lancet 1992; 340: 319-323.

18. Oliver JJ, Webb DJ: Noninvasive assessment of arterial stiffness and risk of atherosclerotic events. Arterioscler Thrombs Vasc Biol 2003; 23: 554-566. 
19. Soga J, Nakamura S, Nishioka K, et al.: Relationship between augmentation index and flow-mediated vasodilation in the brachial artery. Hypertens Res 2008; 31: 1293-1298.

20. Ross R: Atherosclerosis-an inflammatory disease. N Engl J Med 1999; 340: 115-126.

21. Libby P, Ridker PM, Maseri A: Inflammation and atherosclerosis. Circulation 2002; 105: 1135-1143.

22. Kondo T, Hirose M, Kageyama K: Roles of oxidative stress and redox regulation in atherosclerosis. J Atheroscler Thromb 2009; 16: 532-538.

23. Booth $\mathrm{AD}$, Wallace $\mathrm{S}$, McEniery $\mathrm{CM}$, et al: Inflammation and arterial stiffness in systemic vasculitis: a model of vascular inflammation. Arthritis Rheum 2004; 50: 581-588.

24. Spence VA, Kennedy G, Belch JJ, Hill A, Khan F: Low-grade inflammation and arterial wave reflection in patients with chronic fatigue syndrome. Clin Sci (Lond) 2008; 114: 561-566.

25. Klingbeil AU, John S, Schneider MP, Jacobi J, Weidinger G, Schmieder RE: AT1-receptor blockade improves augmentation index: a double-blind, randomized, controlled study. J Hypertens 2002; 20: 2423-2428.

26. Murgo JP, Westerhof N, Giolma JP, Altobelli SA: Aortic input impedance in normal man: relationship to pressure wave forms. Circulation 1980; 62: 105116.

27. Kinlay S, Schwartz GG, Olsson AG, et al.: Myocardial Ischemia Reduction with Aggressive Cholesterol Lowering Study Investigators : High-dose atorvastatin enhances the decline in inflammatory markers in patients with acute coronary syndromes in the MIRACL study. Circulation 2003; 108: 15601566.

28. Hirooka Y, Kimura Y, Sagara Y, Ito K, Sunagawa K: Effects of valsartan or amlodipine on endothelial function and oxidative stress after one year followup in patients with essential hypertension. Clin Exp Hypertens 2008; 3: 267-276.

(Received, February 2, 2012)

(Accepted, April 23, 2012) 